\title{
Fluidity in an orthodontic practice. Why, for who, how?
}

\section{P. Race}

\author{
Designer/Graphic Designer
}

ABSTRACT

The search for fluidity in an orthodontic practice must be an imperative axis of reflection. Fluidity is a quality that must be targeted because it is synonymous with efficiency and comfort and, consequently, profitability.

This profitability is not exclusively financial. It is also at the level of the human. It allows each member of the team to bring the best of himself in his function. And when a team is successful, patients feel it. A satisfied patient is the best advertisement a practice can have. Why deprive yourself?

\section{KEYWORDS}

Layout, ergonomics, fluidity, circulation, profitability, communication

\begin{abstract}
"The shortest path from one point to another is a straight line, as long as both points are already facing each other."

-Pierre Dac

This comment may seem totally absurd, but it is actually far from it.

In fact, it shows that common sense is sometimes quite far removed from reality.

\section{What are the areas in which fluidity must guide our thought processes?}

There are three major areas:

- Circulation

- Communication

- Ergonomics

\section{How to contemplate fluidity?}

One must embrace one's fatigue and conserve one's actions.

The questions a practitioner should ask are

- How to make use of his or her time in the office?

- How to effectively communicate with both staff and patients.

- How to avoid posture problems while sitting down.

\section{Why must we strive to achieve maximum fluidity?}

The main reasons are

- $\quad$ To save time.

\section{Address for correspondence:}

Philip Race - 72 avenue de la Paix - 92320 Châtillon agence. 
- To reduce the number of actions to be taken.

- To make ourselves more comfortable.

- To ease communication.

\section{Who will profit from this fluidity?}

Everyone in the office benefits:

\section{Circulation Fluidity}

Circulation must be designed according to the layout of different spaces and by extension the different processes that take place in a dental office.

Together, these separate spaces must complement each other and therefore function like a molecule. Each atom has a specific function which is tied to several other functions. Molecules can be rather large or complex, but they all have common points.

The first functional location is patients' entrance. The entrance must have both technical and psychological characteristics.

Patients in an orthodontist's office have specific attributes that must be taken into account.

A mother entering with her stroller must be able to open a door without being hampered by overly complicated doors.

A teenager traveling by scooter must be able to leave his or her vehicle near the entrance to avoid damaging the property.

A grandfather who accompanies his grandson must be able to pass by a distracted teenager in the hallway, who has his phone stuck to his ear, without being jostled.
- The practitioner can manage his or her time.

- Staff will not waste time moving back and forth performing various useless or superfluous tasks.

- Patients will be effectively managed without any misunderstanding.

This entrance must therefore facilitate as much as circulation as possible and avoid any bottlenecks (Photos 1 and 2). In this way, it has similar functions as highway toll gates.

Wednesday afternoons are a bit like the famous cross-over of late July and early August. The difference being that the number of toll booths can be modified whereas the dimensions of the office's entrance cannot be easily adapted.

From this entrance, the reception area must be clearly defined and identifiable from any direction. Patients who enter must know where to go without having to wonder about it.

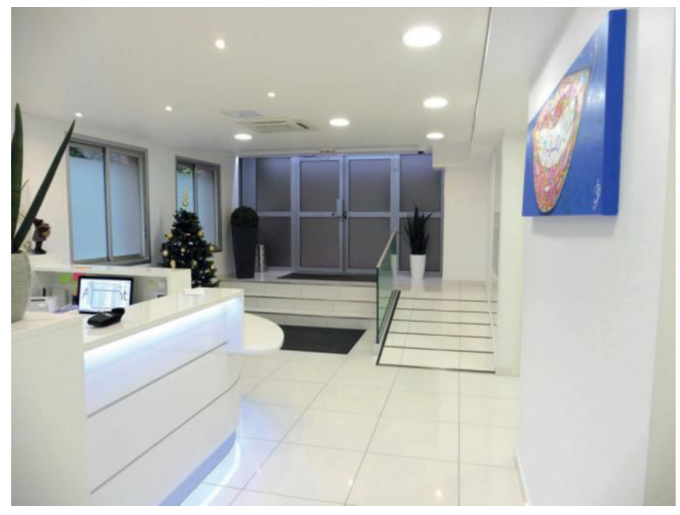

Figure 1

A wide and bright entrance. 


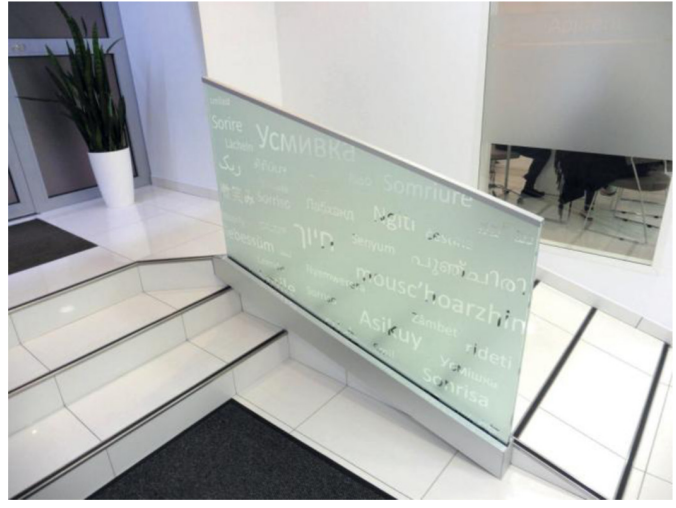

Figure 2

A ramp for handicapped

and disabled persons as well as persons with strollers.

They will either pass through a personalized reception area with dedicated staff who will receive, inform, and direct them, or if they are already receiving treatment, and the office is so equipped, they can register themselves using the digital self-service terminal without having to otherwise announce their arrival (Photos 3 and 4).

In both cases, the physical reception point or digital terminal, must be immediately visible and identifiable as a mandatory checkpoint for any patient entering the office.

If this is not done the following could result: reduced circulation of patients, ineffective management of staff, and lack of fluidity from the primary function.

The reception area can incorporate a second function: the secretary's desk.

This function is multifaceted. It can be used for the following:

- The administrative management of the office.

- The reception of patients and/or persons accompanying them to deal with their paperwork.

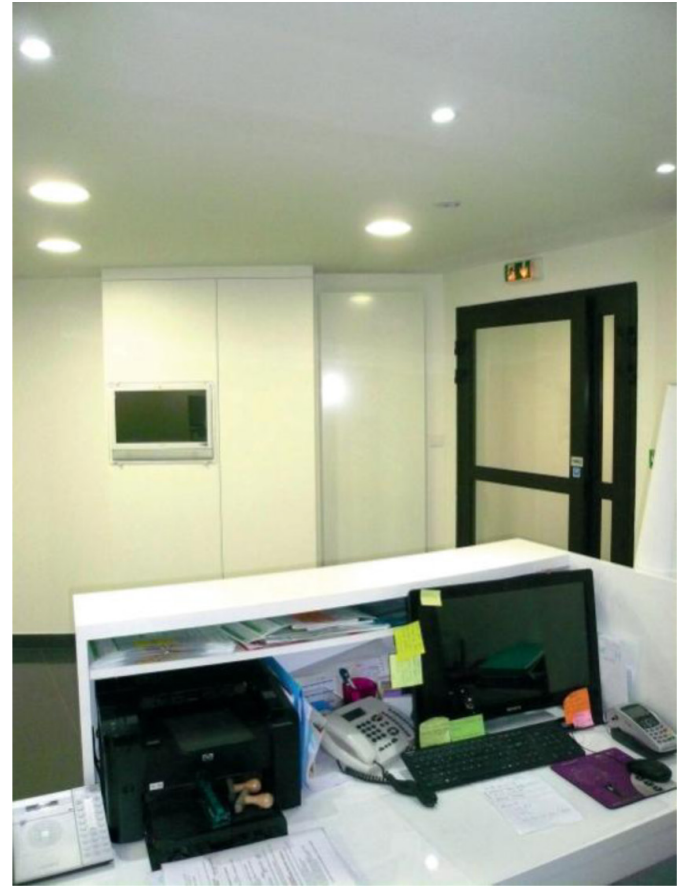

Figure 3

A digital reception terminal to complement the physical reception.

- The post at which treatment plans are explained by an assistant consultant.

These additional purposes must not hamper the major purpose, which is receiving patients and guiding them around the office.

While arranging this station, priority must be given to the length of time to be allocated on a patient-to-patient basis.

A patient who wishes to announce his or her arrival will remain standing.

A patient who wishes to deal with administrative matters must take a seat, but he or she should not be stuck at the reception point for too long.

A patient to whom treatment details are being explained must be comfortably seated. In this instance, there 


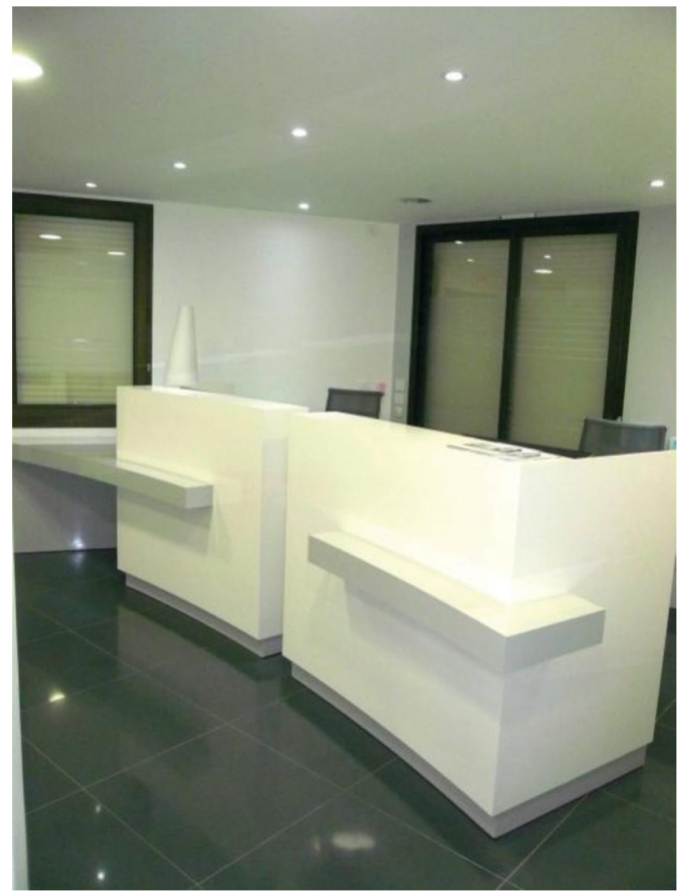

Figure 4

Reception equipment that automatically positions patients.

will be a dedicated space for this type of service, and often, a member of staff will be assigned to this function (Photo 5).

Finally, a telephone reception must be included, which is likely to slow down the receptive capacity of a wellthought-out reception area.

It is therefore equally important to have a clear understanding of how the phone system works when operated by the reception staff, which tends to be the case in many practices.

In addition to all these features, one must take into account the regulations regarding persons with reduced mobility. At these stations, a shelf should be provided, allowing a person in a wheelchair a place to write if necessary (Photos 6, 7, and 8).

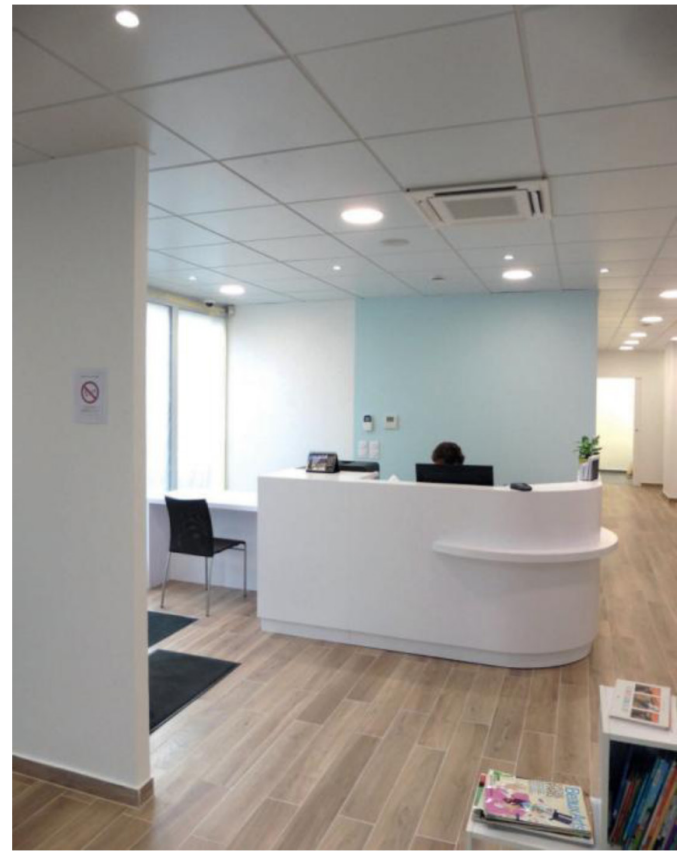

Figure 5

Differentiated reception modes seated/standing.

When designing the placement of these stations, the dynamic and static areas must be clearly identified. The dynamic areas are where patients move about or stays only a short while. Whereas the static areas are where patients spend a relatively longer period of time and therefore should not block the passageway.

If these conditions are respected, the fluidity of the reception process will improve, while various tasks are being accomplished (Photo 9).

Patients then go to the waiting area.

It must be easily identifiable and may be open or enclosed with one or multiple access points (Photo 10).

Its layout will mostly depend on the practitioner's method of operation.

The type of patient is important: adults, adolescents, and/or children. 


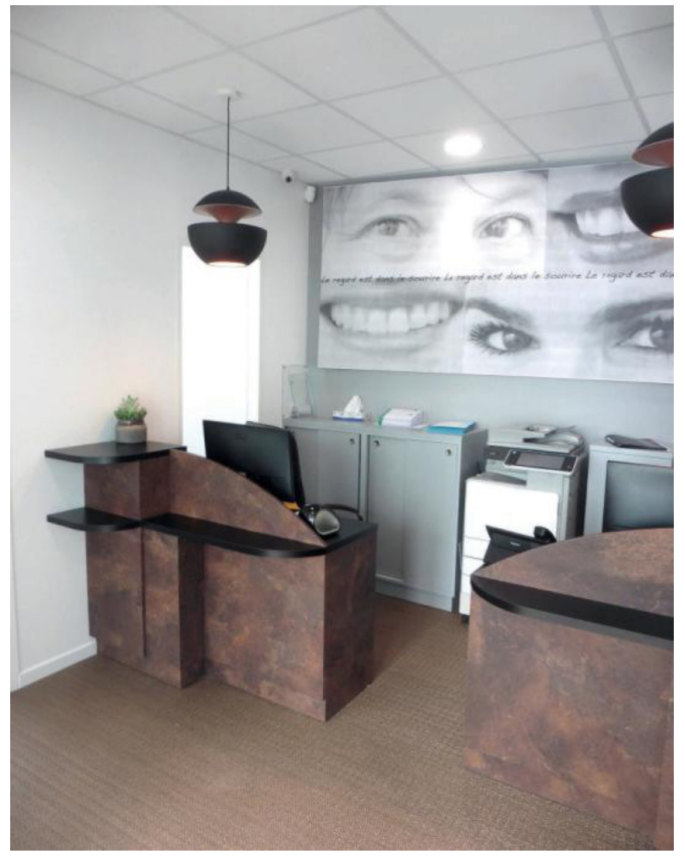

Figure 6

Easy circulation of staff.

In the case of adolescents or children, the adult companion must also be accommodated for. Should they escort the patients into the treatment areas or should they remain in the waiting area?

This point is also important because consideration must be given to the waiting area (Photos 11 and 12).

If the adult companions stay in the waiting area, it is understood that patients will return to this space to meet them after their treatment is completed.

There is therefore a backward and forward flow into this space.

If the adult companions escort the patients into the treatment room, circulation can then be linear. There is no need to return to the waiting area because there will be no one there to meet.

If an office has an exclusively adult patient population, this is no longer an
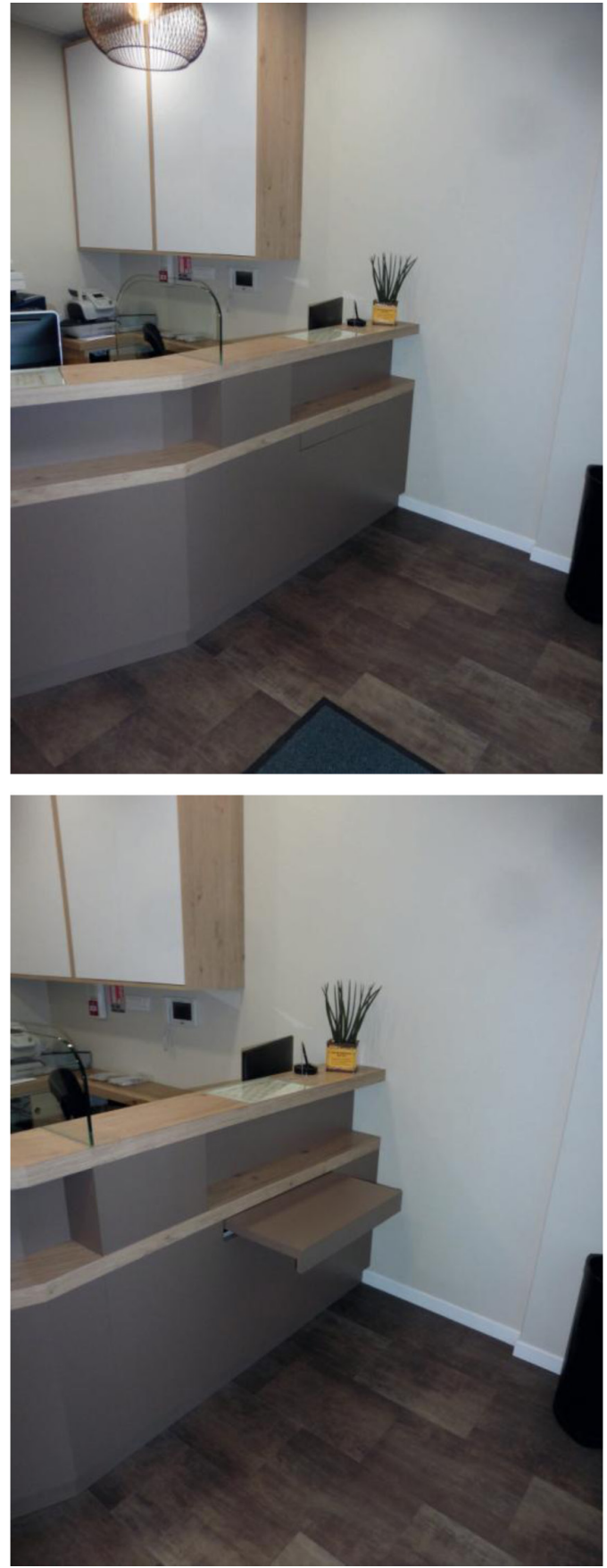

Figure $7-8$

$A$ response to the adapted regulations.

issue. The majority of older patients come alone, and as such, because of their autonomy, they can avoid passing 


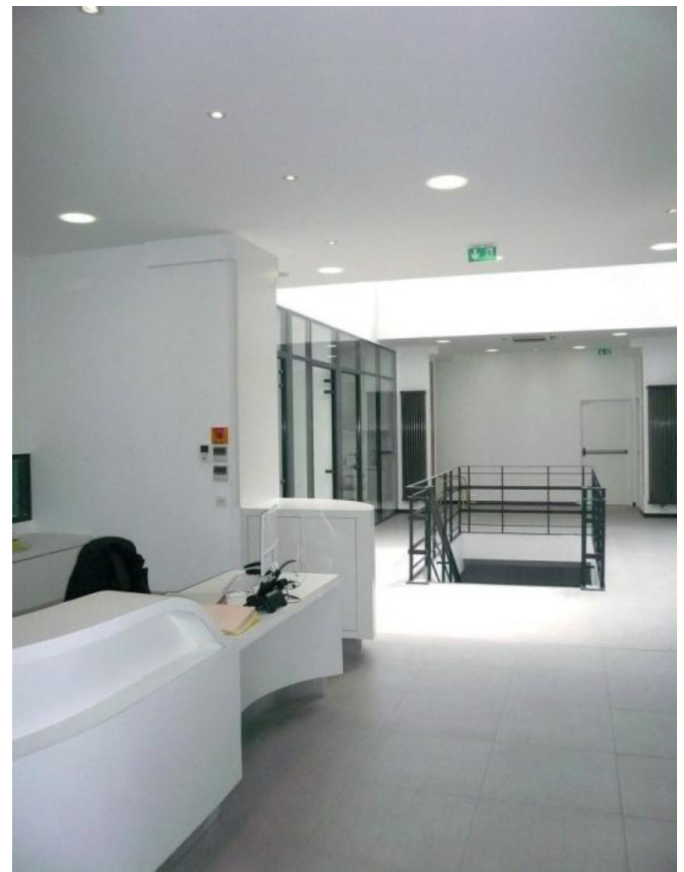

Figure 9

A station with multiple functions.

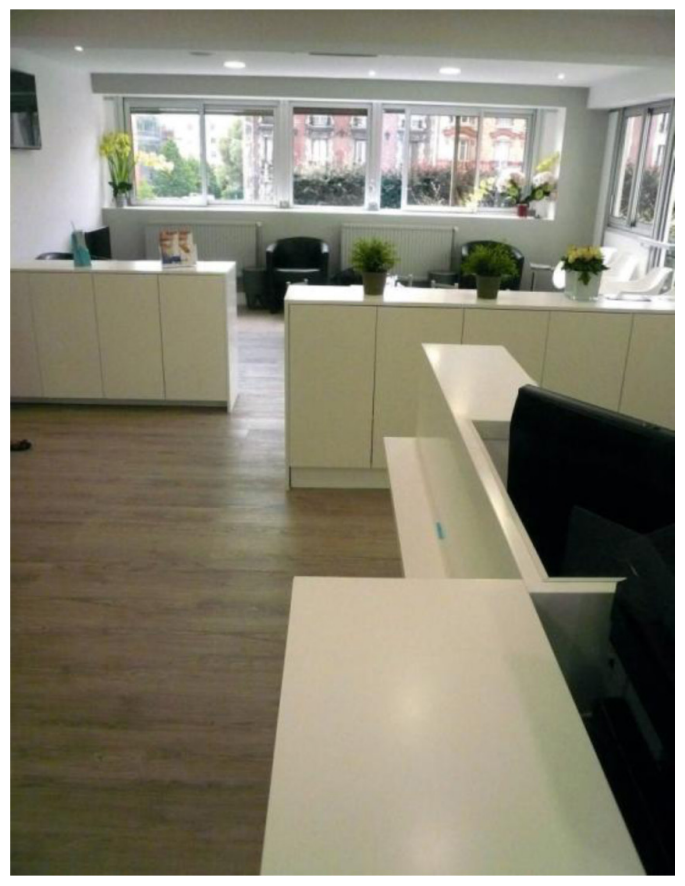

Figure 10

Option open space with a view of the waiting area. through the waiting area after treatment.

The positioning of the waiting area, number of access points, and clearance height (low enough so it can be reached) will therefore be the determining factors in increasing fluidity.

This space, whether open or enclosed, must be close to the entrance and reception.

It must be close enough to the entrance to avoid a substantial patient flow in the office, particularly a crossflow in medical areas, and close enough to the reception to facilitate administrative services such as making appointments, payment, and signing various documents.

These two functional areas-the reception and waiting room-as well as the restrooms (when accessible by patients) and brushing stations should all be located in the first segment of the office.

These spaces are accessible to everyone who enters the office door.

As far as possible, these spaces should be maximally secured to prevent patients from venturing further into the office toward the more technical areas.

The aim is to facilitate the flow (movement of persons) and especially to be able to distinguish the staff from patients in medical areas.

By going further into the office, we are sure to find other areas before arriving in the treatment room.

There are three main areas: imaging, radiology, and consultation.

When the patient population is made up of children and adolescents, consultations unavoidably require the presence of their adult companions. 


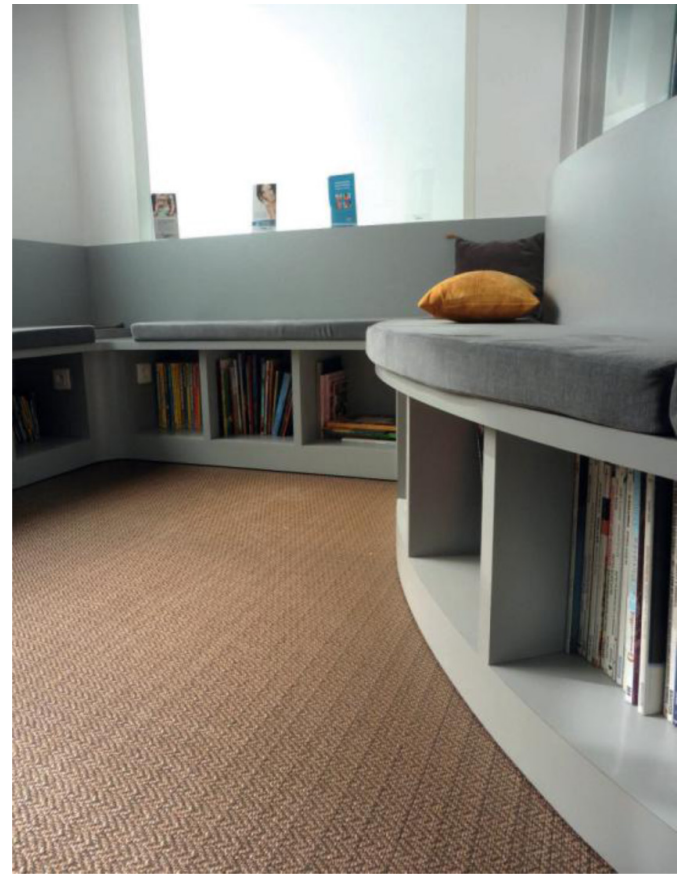

Figure 11

Functional seating.

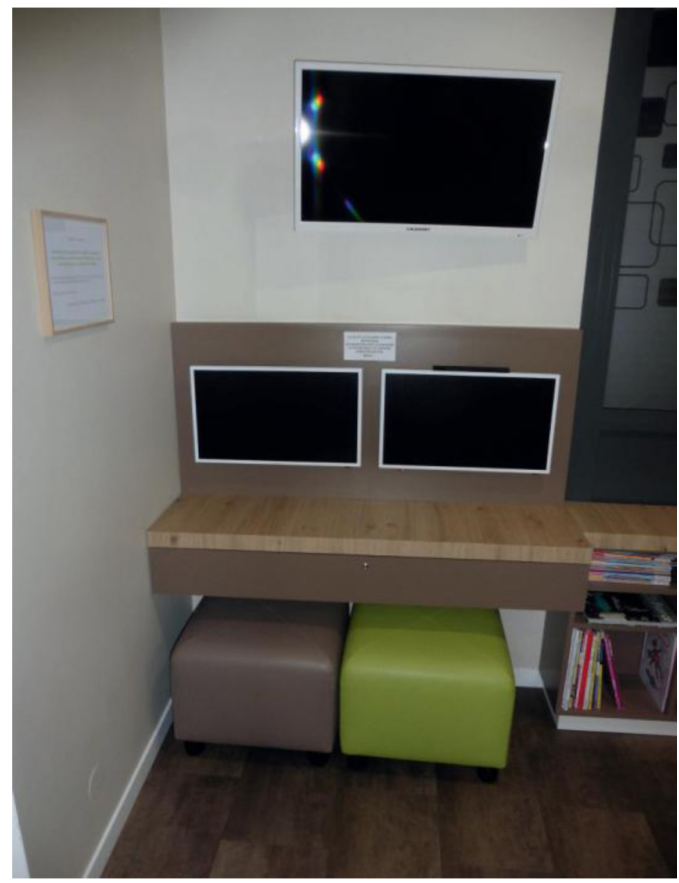

Figure 12

Fun spaces for all ages.
The consultation room must therefore be close to the waiting area because it may generate significant traffic owing to the fact that patients being called for consultation are coming from the waiting area.

Consultation is also the place where assessments are conducted for ongoing treatment.

It must be large enough to receive several people at once and designed to place them in an ideal position so that they would be readily receptive to the practitioner.

Everyone must be distributed across spaces without hampering the circulation (Photos 13, 14, and 15).

The companion's seats must be easily accessible without them having to cross the space, thereby increasing the risk of getting in the way of the staff and other patients.

Similarly, patients must be able to access chairs as easily and as directly as possible.

The practitioner needs to function in his or her own space, relative to that of the adult companion, without the latter being in the way.

This space may have yet another function: treating emergency cases.

Its proximity to the waiting area, and by extension the reception area, makes it possible to treat a patient in an emergency without necessarily having to pass through the waiting area.

The patient therefore will not have to pass "in front of everyone," thus avoiding panic among the other patients.

The imaging and radiology rooms are not highly aseptic. These two functions can be handled independently of treatment, but they can also be combined with it. 

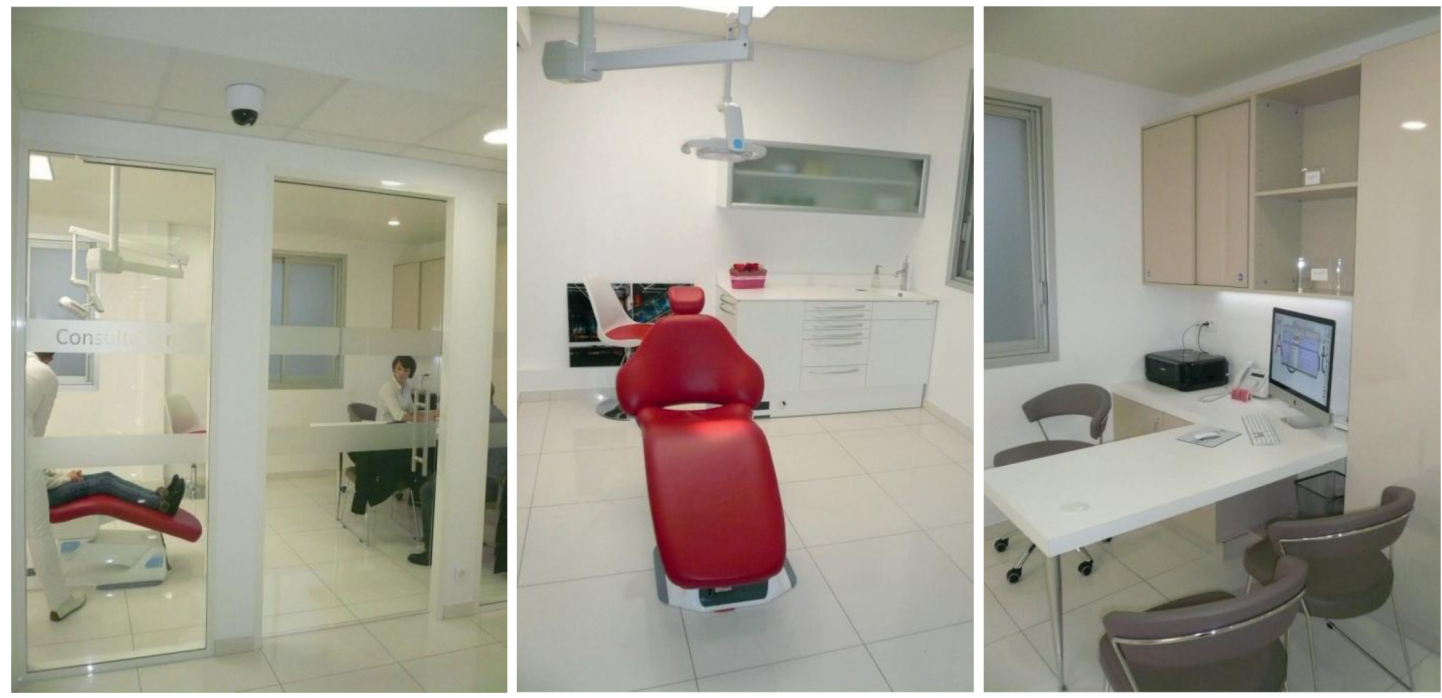

Figures 13-15

A consultation area where several persons can be received, and care provided if necessary.

Their positions in the office should therefore be close to the administrative areas, but not too far removed from the treatment area.

Once treatment is started, one of the possibilities offered to the practitioner is to assess the patient from the first appointment. In this case, taking a panoramic X-ray, an impression, and photographs will be the first actions performed. Hence, there is a need to position the required functions close to the consultation room.

However, radiology being a medical procedure is reserved for the use of the practitioner only; therefore, the practitioner's office should not be placed too far from the radiology room.

If one is to assume that the profitability of the office relies on "the chair," it is imperative that the radiology room is close to the treatment areas.

If there are fewer and shorter journeys between areas, more time can be spent on treatment. This is not necessarily synonymous with a patient "treatment chain." Dealing with a patient in the chair involves care, training, and motivation.

The practitioner must therefore be physically available and compassionate.

As such, he or she must avoid being asked to perform additional tasks.

At last, we have arrived at the treatment area. This is the area where a higher level of cleanliness is crucial.

Additionally, in this section, each patient's movements must be as short and as direct as possible to avoid cross movements as much as possible (Photos 16 and 17).

The three characters are the practitioner, the chairside assistant (for four-handed tasks), and the patient. The patient can be accompanied and, if the practitioner so chooses, the companion may have to assist the patient in the procedures.

To ensure optimal respect for both the fluidity of movement and cleanliness of this space, each person's movements must be clearly defined. 

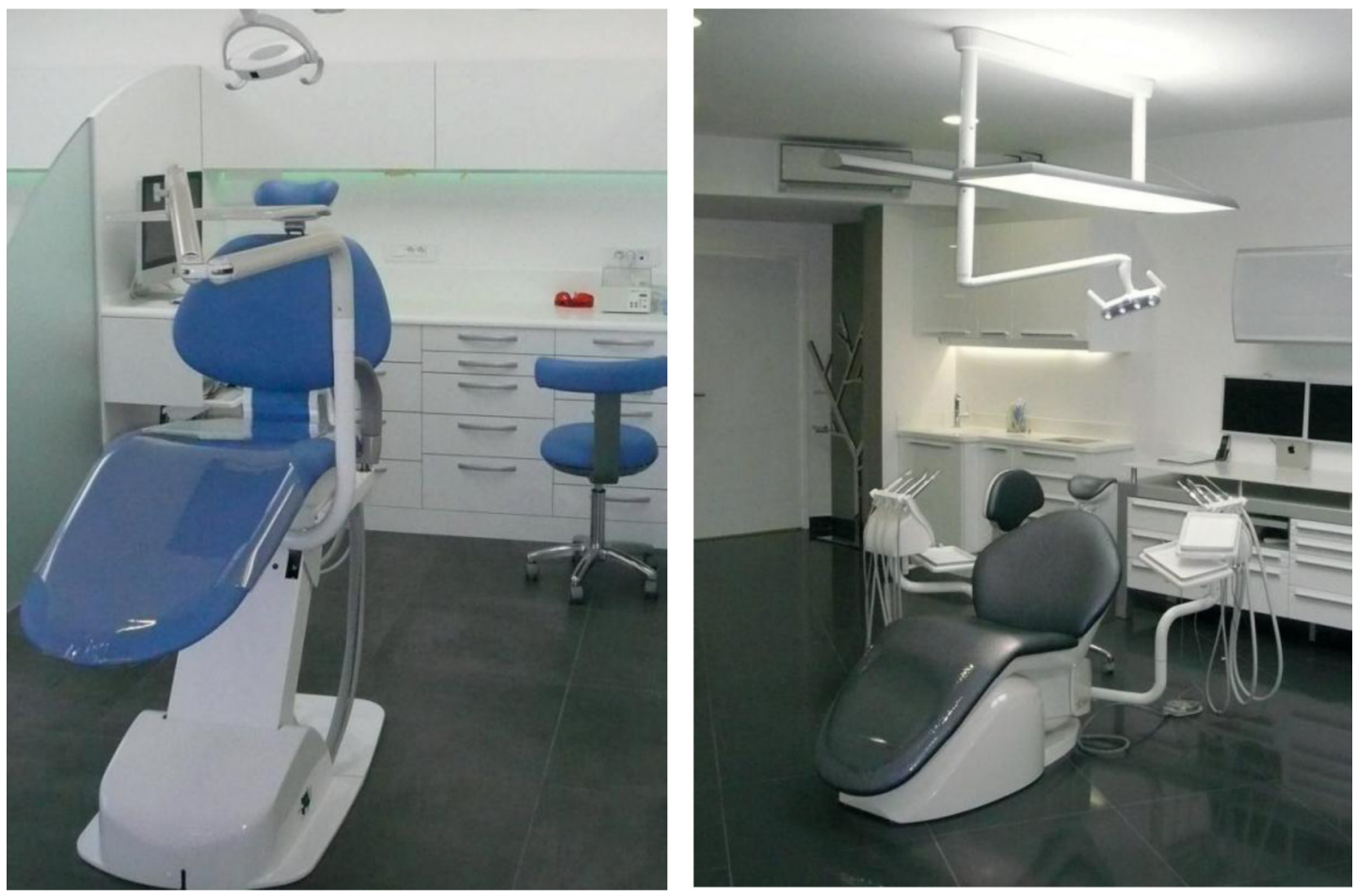

Figures 16-17

Easy access to the chairs.

The practitioner circles the chair in either an anticlockwise ( 9 o'clock) or clockwise (3 o'clock) movement depending on whether he or she is left- or right-handed.

The chairside assistant may assume several positions depending on the type of procedure being performed. He or she may be directly opposite the practitioner on the other side of the patient's head in some cases, but also may be on the same side as the practitioner, further toward the foot of the chair in other cases.

Patients must have direct access to the chair from the moment they enter the treatment area.

At all costs, they must avoid crossing the practitioner's space or having to go around the chair to get onto it.

If the practitioner has a desk in this space, he or she must be able to get to it without affecting the flow of movement of the patient or assistant.

At the end of the treatment, the assistant must be able to leave the area without cutting across the patient's or practitioner's circulation (Photos 18, 19, and 20).

The companion, if there is one, must stay as close to the entrance as possible and must never get in the way of the assistant.

It is obvious in this case that at the end of the treatment, the assistant must have the greatest access to the sterilization equipment. He or she constantly strives to keep the area clean for the following patient, and his or her task must be facilitated by having as short a trajectory as possible with as few obstacles as possible so that he or she can reach the necessary materials with 

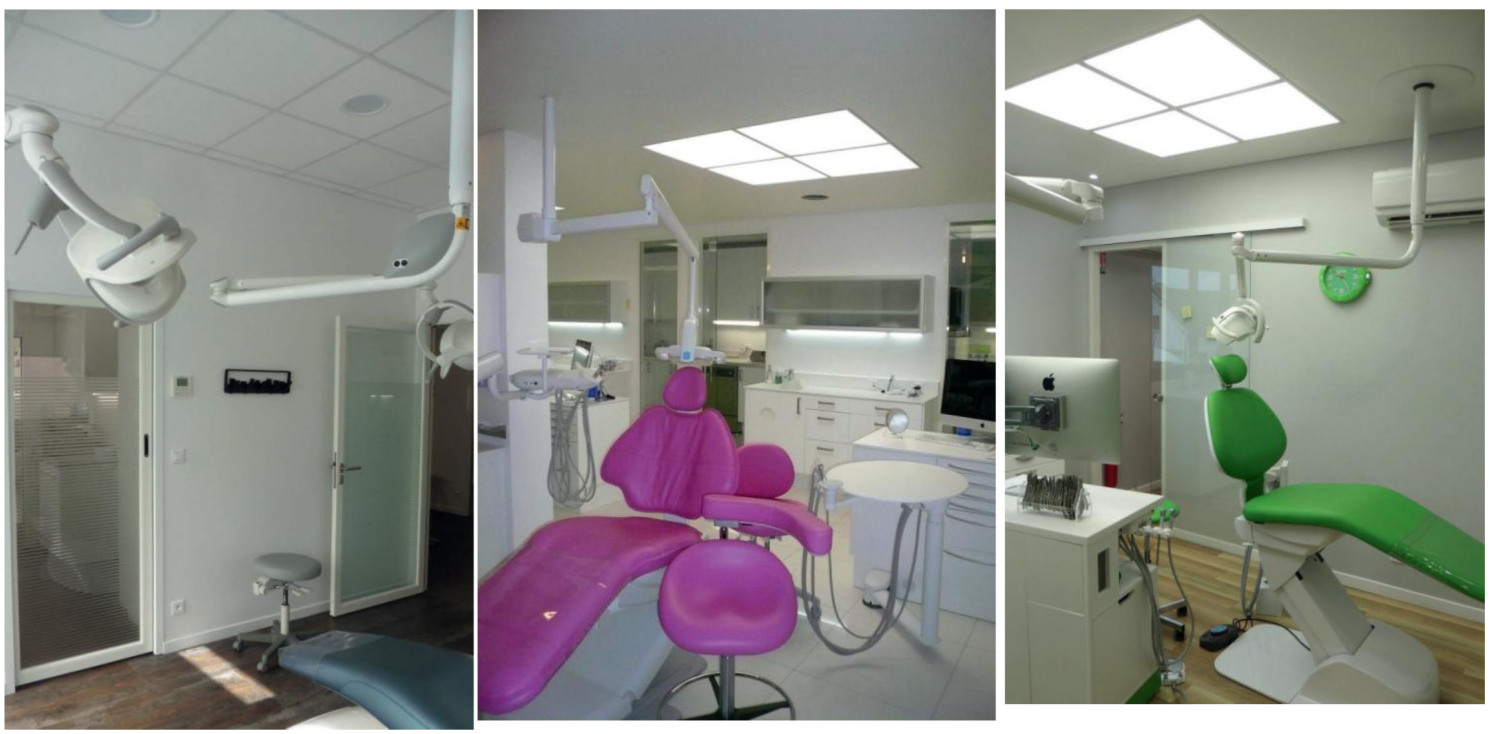

Figures $18-20$

Direct flow of movement between treatment and sterilization areas.

minimal effort. To simplify this purpose, in other words, it would be ideal to have an easily accessible location with direct access to the sterilization equipment with an area to place dirty materials and waste without having to make an extra effort to open a door to access the garbage bin. Easy access to cleaning products should also be available without having to move too far away from the chair and work area for disinfection.

The sterilization and treatment areas must be as adjacent as possible to effectively manage and increase the fluidity of movement between chairs (Photo 21).

What is easy enough to design for two or three chairs becomes much more complicated when the number of chairs increases.

Nevertheless, we must keep the operating criteria in mind when configuring the layout.

There must also be fluidity in the sterilization area.

Not only will it save the assistant's time, but it will also ensure the smooth functioning of waste management, disinfection, and sterilization protocols.

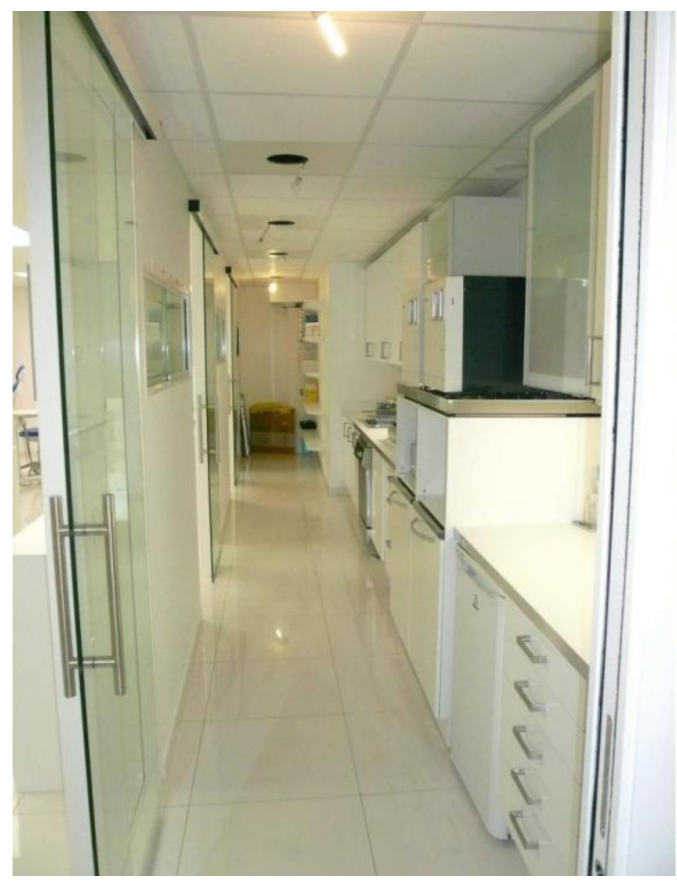

Figure 21

Sterilization following a linear treatment chain. 
The principle is simple. The sterilization chain begins with a set of dirty instruments and different types of waste and ends with sterilized instruments that can be readily identified.

What is unique about the process of sterilization in an orthodontic office is the fact that its treatment cycle varies according to the peaks in activity.

It should be noted that this characteristic of "swelling" can also be applied to the waiting and reception areas.

Sterilization sometimes leads to difficulties due to the large influx of instruments to be treated and the low availability of staff.

The area must therefore be designed in the same way a hydrographer designs a river course with expansion basins in case of floods.

The area that houses the "dirty equipment" must be big enough to store a large volume of materials before they are treated, but storage must be minimal. It is therefore necessary to try to dispose of waste and packages and only store things that have to be disinfected and or sterilized. This section is easy to organize and will lead to greater fluidity. The placement of and access to the bins is of paramount importance. Consequently, the assistant will have fewer doors and drawers to open to throw things away and as a result will be able to free up some time to continue with the rest of the protocol.

Using decontamination basins also aids in expansion. Instruments are not left in the open air waiting to be treated. The disinfection machines are located after these basins. They are gradually filled as the activity in the chair progresses, and the cycle is launched when they are full.
Whenever possible, disinfection should be performed close to the bagging area. Once the instruments have been dried, the assistant can put them into bags without having to carry them too far. This step requires a worktop surface but only temporarily. It is therefore a practical solution to design retractable worktops similar to sliding shelves. When they are not in use, they can be retracted into the furniture (Photos 22 and 23). As such, they do not hamper circulation and sterilization, which brings us back to the concept of fluidity.

In general, the layout must factor in the importance of time. All actions do not take place simultaneously. An area can be free at time $\mathrm{H}$ and occupied at times $\mathrm{H}$ and $\mathrm{N}$. To ensure fluidity, the operational protocols must be specified from inception.

Once the sterilization cycle is complete, the "clean" materials must be stored as close to the entrance as possible but also very far from the "dirty" ones.

If the sterilization area is adjacent to the treatment area, a "pass-through system" or sliding furniture is a viable solution.

Special care should be taken not to allow the "clean" materials near the "dirty" ones and vice versa.

Movements are imperatively dissociated, and this differentiation can be described in terms of volume:

The "dirty" area having fewer furniture and the "clean" area having more furniture. Thus, the "clean" sliding furniture will be above the low furniture "bins."

Sterilization may have a complementary function of supplying consumables (Photo 24). 

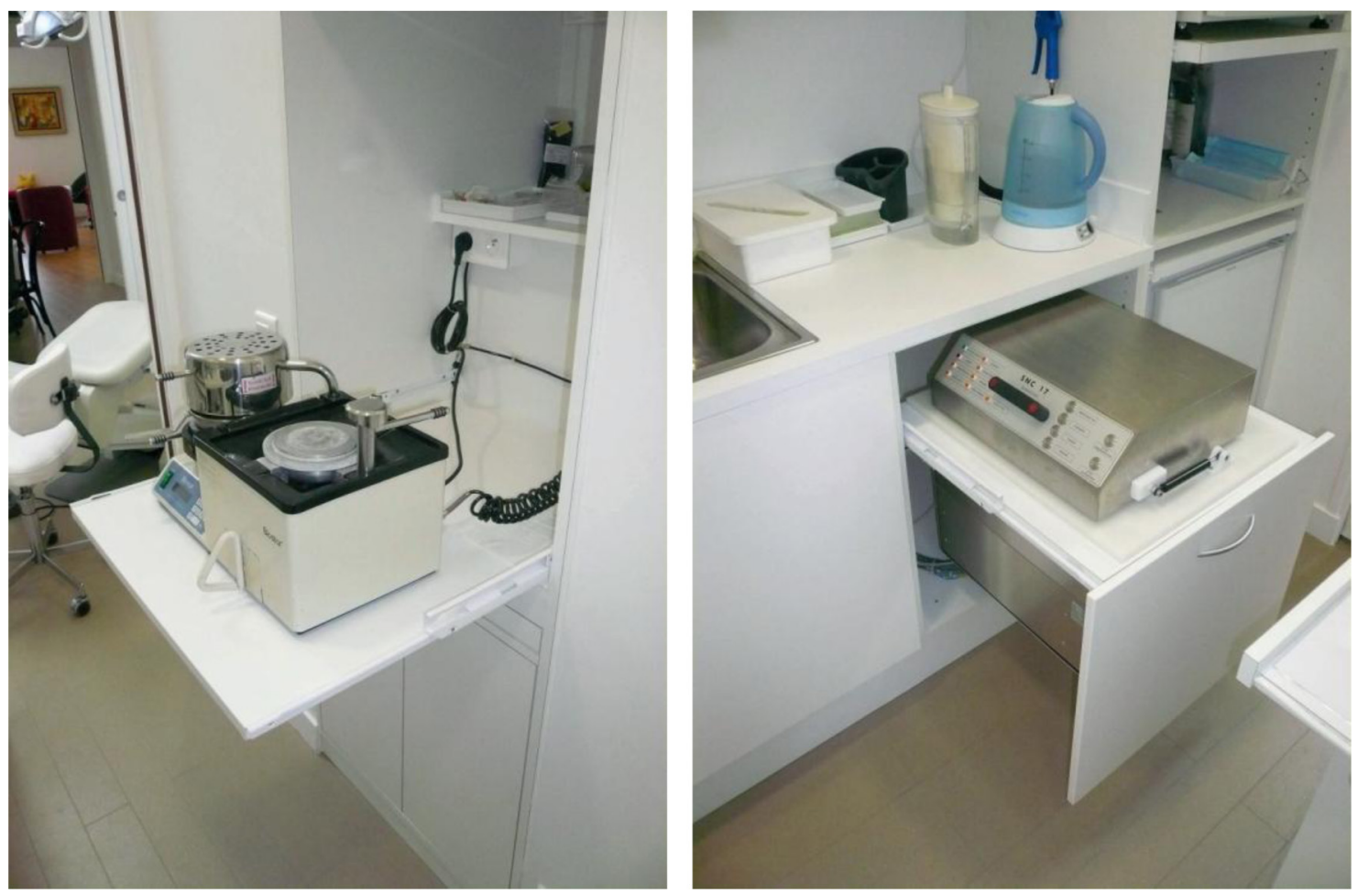

Figures 22-23

Adaptable work surfaces.

To ensure the fluid management of consumables, the office must be equipped with three degrees of storage:

- Long-term stock. This stock is used for receiving orders and can be placed far from the area where the consumables are being used and also close to the delivery access. The long-term stock is used to gauge the requirement for the replenishment of the medium-term stock.

- Medium-term stock. This is the intermediate stock that has to be stored close to the areas where daily consumables are used. These consumables may be needed in treatment areas as well as administrative spaces. Therefore, they can be split into two separate stocks.
- $\quad$ Short-term stock. They can be found in areas where consumables are used, usually in the furniture in treatment areas or secretary's office.

There are other functions that can improve the fluidity of the office space:

- The laboratory

- The assistants' station

- Staff break area

The laboratory has features in common with the sterilization area. It must be situated close to the treatment areas if it does not function independently, and the chairside assistant must have quick access to it.

If the space is to be used by a prosthetist, it can be completely independent. 


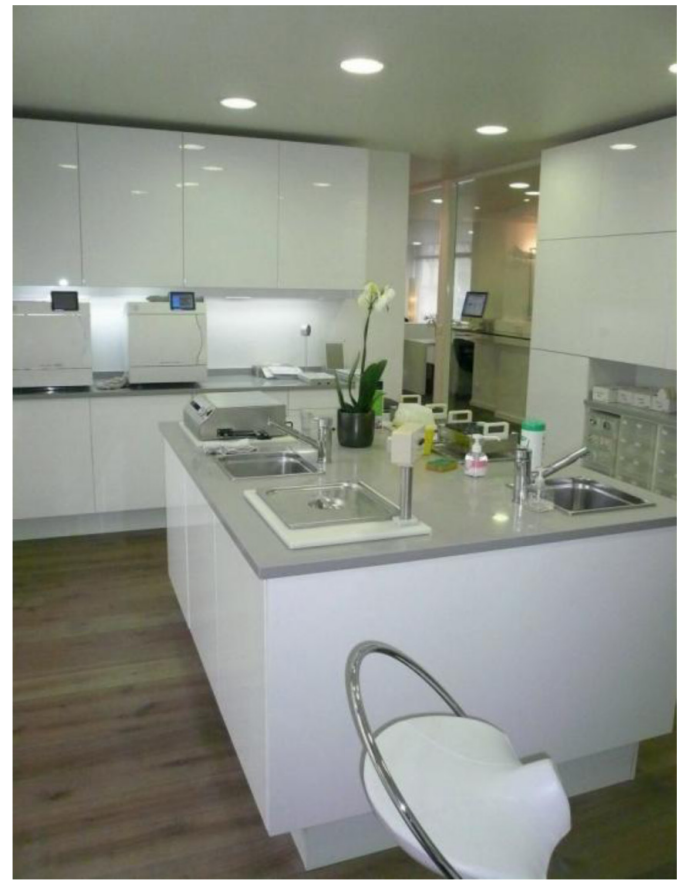

Figure 24

Sterilization and storage of consumable supplies near the treatment area.

However, procedures such as grinding can be carried out in the chair so that the practitioner would not have to go to the laboratory.
In this case, a suction attachment can be included in the practitioner's space and should be placed near the chair. It can be discreet, perhaps hidden in a drawer where the grinding motor can also be stored. The foot pedal is positioned in the base board. The practitioner remains at his or her post where he or she is able to carry out final improvements.

The assistant's stations are areas where patients can receive explanations or else they may be used to ease the burden on the reception area. They must be situated on the path between the chairs, waiting area, and reception. The assistant can provide patients with pertinent information such as follow-up treatment and making appointments.

Since these stations are close to the waiting area, if the assistant needs to communicate with a patient's companion, they can be easily located without increasing the traffic in the walkways.

It is strongly advised that to avoid seated stations, discussions should take place while standing up (Photos 25, 26, and 27).
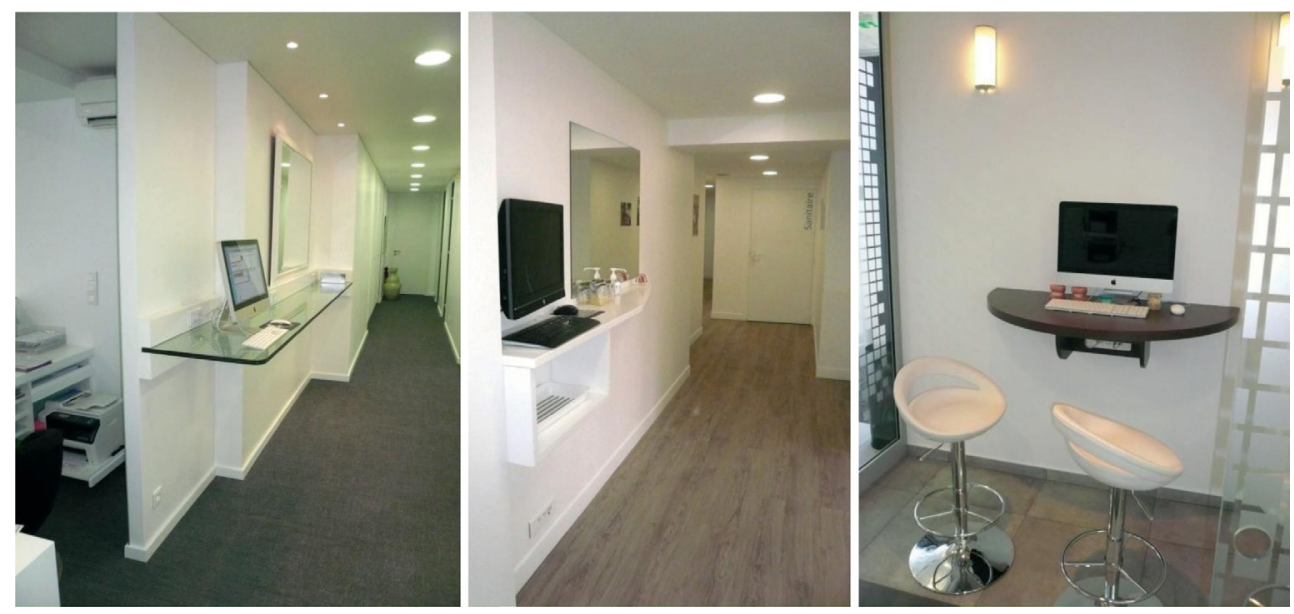

Figures 25-27

Assistants' stations to relieve the reception and treatment areas. 
The staff break area is governed by some of the provisions of the Labor Code.

The changing room should have fluidity as all members of staff arrive at approximately the same time (Photos $28,29,30$, and 31).

There is therefore a risk of congestion. It is essential to find planning solutions that will allow the staff to change without (too much) embarrassment while respecting each person's modesty.

If the staff is mixed, two changing rooms should be provided. Some offices prefer to distinguish the staff's changing room from that of the practitioner.

In the office layout diagram, the placement of this function differs depending on whether it is a simple changing room or an area equipped with a kitchenette.

In the first case, its position, whether toward the entrance or toward the back of the office, is of little important because this space is mostly used outside the presence range of the patients.

In the second case, it will be better positioned toward the back of the office. In this way, patients will not be able to see when a staff member or the practitioner goes there to have coffee.

Generally, one should try to separate the patients' movements from those of the medical staff.

The fluidity of the office is dependent upon the practitioner's time management skills, the practice of which will be applied to all staff members.
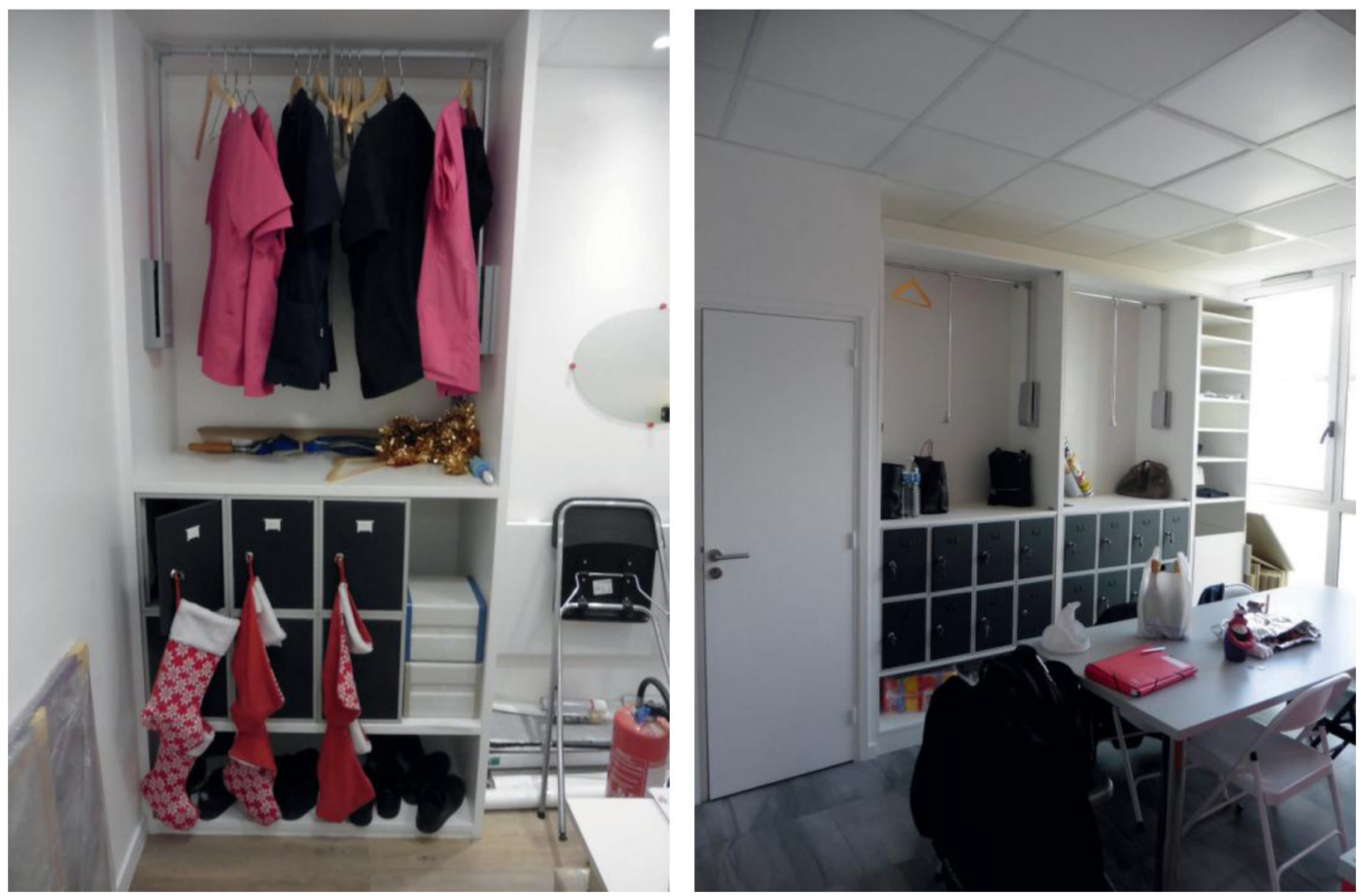

Figures 28-29

Changing rooms specially modified for the staff. 

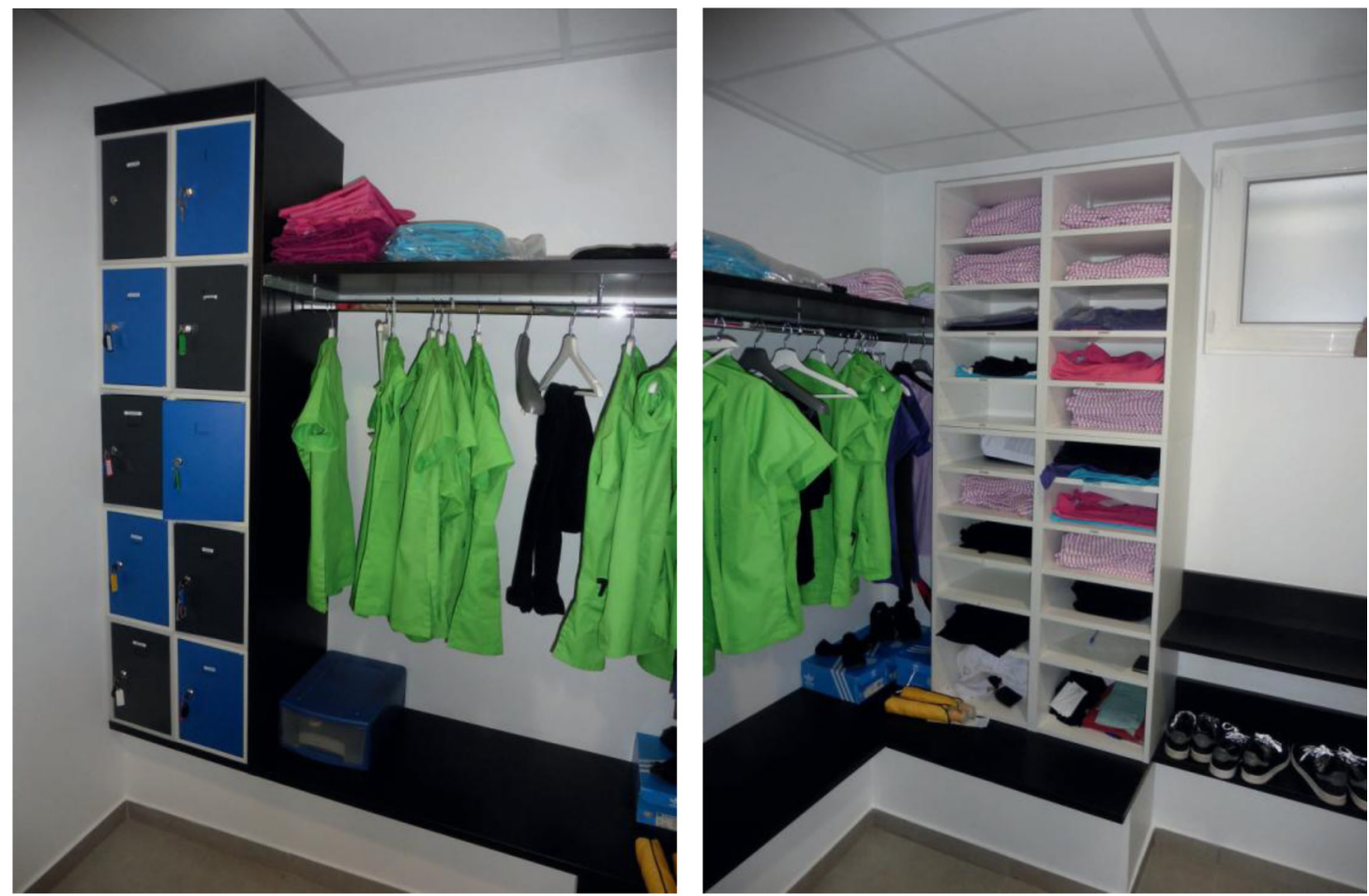

Figures 30-31

Changing rooms specially modified for the staff.

The practitioner should avoid going around to the patients and should only meet them if he or she so desires or if there is a requirement.

This does not make him or her inhumane, but is necessary for effective time management.

It will not prevent the practitioner from getting to know the name of his or her patient's (who just entered sixth grade) little brother's dog, but it will prevent him or her from being detained by the bothersome patient who comes to explain his or her colleague charges 3\% less and that it is ludicrous to charge such steep amount for treatment.

Fluidity in communication is also important with regard to circulating information among members of staff, between the staff and practitioner, or with other practitioners.
Communication can be either oral or visual.

The receptionist must be able to inform the practitioner if a patient has a question or a problem that only the practitioner can solve. He or she must be able to reach the practitioner easily and quickly and explain why his or her presence is required. There are several ways this can be achieved: an internal telephone system, internal use of a telephone network, and use of a video network, which can open a window on the practitioner's computer screen.

It is also possible to create a direct view between the treatment area and reception if the office is not too wide, but beware: this is a two-way view.

The practitioner may also require the services of a chairside assistant or secretary for a specific administrative 
matter and may not be able to leave his or her workstation.

The basic call would consist of using a bell to indicate that someone is needed. However, where should the bell be rung, how will the person being summoned know where to go or whether her or she needs to bring a file or an instrument or simply to complement the practitioner's two hands?

Therefore, alternate means that provide more details should be considered to improve efficiency.

The ideal situation is to meet the staff right where the practitioner is and to be able to express his or her need. By combining this option with the staff's ability to answer phone calls wherever they are, the most suitable system is the use of a telephone with an intercom function.

This system involves the placement of telephone stations in treatment areas, where the practitioner could make a request without having to move physically.

Beware: this system will lead to increased glove consumption.

If the practitioner thinks that he or she does not need to be able to contact the staff for precise requests, a simple call button would suffice. However, if the practice is big and the number of chairs is substantial, the practitioner should let the staff know where he or she is. $\mathrm{He}$ or she should therefore ring the bell, which is the most effective signal, along with visual indicators, e.g., colored lamps, to specify where the call is coming from. This is the equivalent of hotel room service. These visual cues may be close to the bell or above the access doors to the treatment area. In a space with several chairs, a single indicator will suffice.
This procedure allows staff to get there quickly without wasting time; this again stresses the importance of fluidity. They go directly where they are needed.

We have seen the physical solutions to communication issues that arise during the business activity of the practice.

We must also approach communication in a more general way: this concerns the operational mode of the practice.

In the industry, there are facilitators, who are persons without any particular assignment except the fact that they communicate with everyone outside the hierarchical structure. They circulate information that can be used to improve services or everyone's working conditions and ensure that this information is passed on to those incumbents who can change the way they work.

Unfortunately, this position does not exist in offices, which is a pity. As a result, a communication exchange process must be established between the staff and practitioner.

The exchanges are two-way. The practitioner encourages the staff to adapt to his or her methodology. He or she is the captain of the ship and the face of and the brains behind the business.

However, he or she cannot be everywhere. It is therefore very important for him or her to be able to receive information.

An intelligent secretary will know better than anyone how to arrange his or her workstation. He or she will be able to submit ideas regarding the ergonomics of their station and suggest improvements in the reception process that the practitioner can utilize. 
The same goes for sterilization and laboratory functions. The assistants can help improve the office's organization by making proposals regarding the constitution of trays and management of stocks among other things.

This implies that the staff is involved in the life of the practice. So basically, the practitioner's major role is to motivate his or her team.

Going back to the office layout, as long as space permits, an area must be reserved for staff meetings (Photos 32 and 33).

It is not necessary to have a dedicated space for these meetings. The waiting area or the staff break area may be used for this purpose.

The meetings will take place outside of working hours; temporary accommodations are easy to plan.
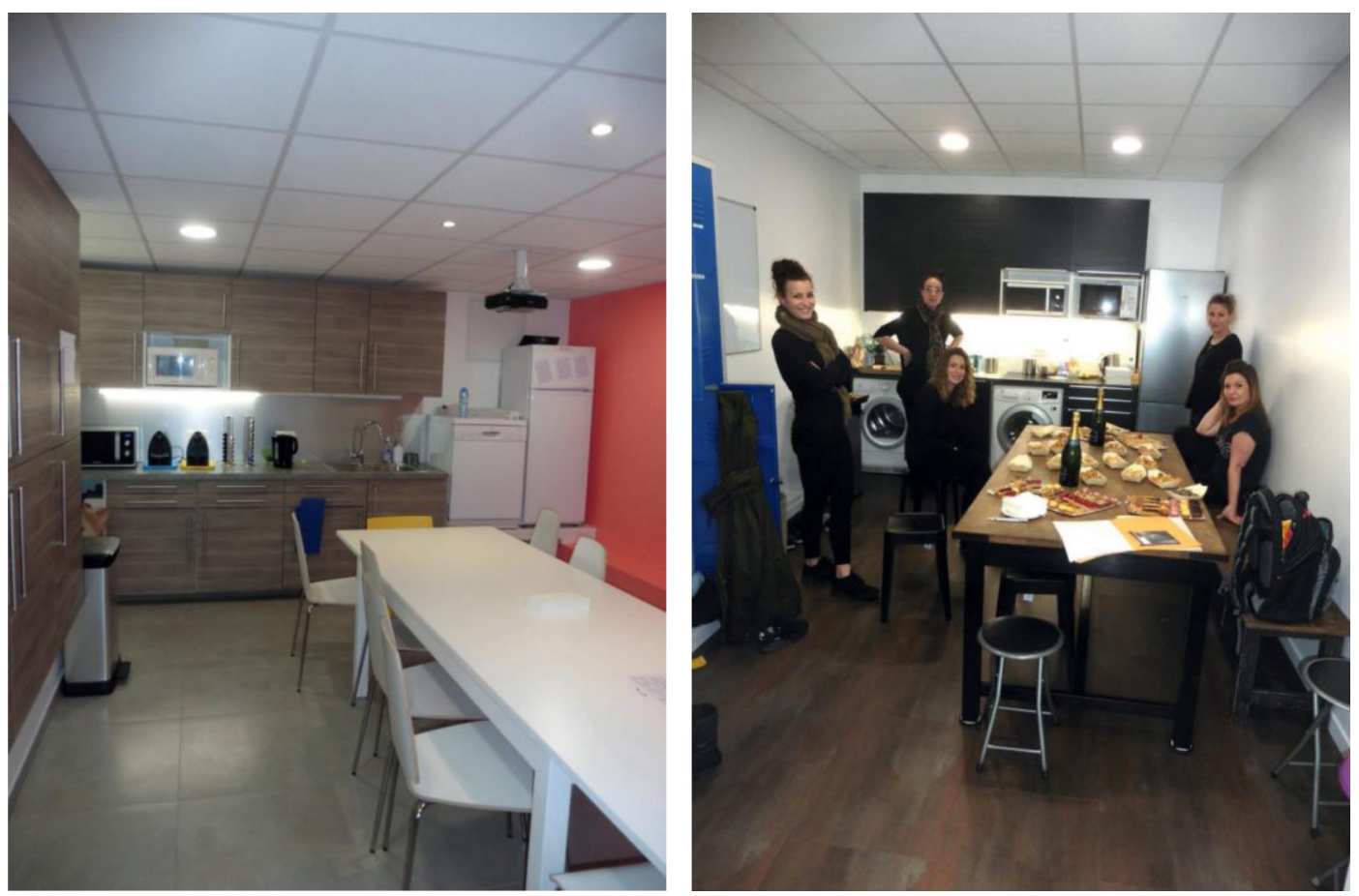

Figures 32-33

Spaces to meet and encourage socialization among team members.
If all these elements are included, communication will be fluid and more effective.

Each member of the team will know his or her role and bring his or her expertise while the captain carefully steers the ship.

\section{Ergonomic Fluidity}

The principle always remains the same: make minimal movements.

However, we must add: avoid straining yourself.

The functions mainly concerned with ergonomics are

- Chair work (practitioner and assistant)

- Secretary's work (secretary)

- Sterilization work (assistant)

- Lab work (assistant) 
As much as extended movements through the office are discouraged, so too, on the other scale, spending too much time at a desk should also be avoided.

Taking two steps with a wheeled stool to find an instrument in a drawer shows that the instrument, if it commonly used, is not in its proper place.

The same goes for the treatment area and secretary's desk. If the secretary has to get up to collect a document he or she has just printed, the printer needs to be repositioned.

If an autoclave is placed too high in the sterilization area, this could cause the instruments too fall or an assistant may get burned if he or she mishandles the equipment. Its positioning must be considered in relation to its accessibility, especially if there are numerous sterilization cycles.

The movements of all individuals in the office must be planned to avoid unnecessary shifting, twisting, and bending.

Optimizing the ergonomics of each station will not only provide fluidity in the management of tasks but also avoid body aches.

This will also lead to gains in fluidity because absenteeism is minimized due to better physical care; a full team is certainly more efficient than just a part of a team.

Through the points highlighted here, it can be noted that fluidity is a sine qua non for the smooth functioning of the practice. It is therefore important to strive toward achieving this quality despite all the limitations imposed by the building.

With the exception of construction commissions, it is quite difficult to achieve the perfect project. Perfection does not exist; you must ask yourself key questions while designing the project and identify areas to prioritize that will improve the success of the practice and make the team as efficient as possible.

A practitioner breathes life into his or her practice, as he or she is the one who holds the rudder.

Happy sailing...

Conflict of interest: The author has no conflict of interest. 